\title{
Abraham and the tortoise: eleatic variations on Fear and Trembling
}

\section{[Abramo e la tartaruga: ariazioni eleatiche su Timore e tremore]}

\author{
Igor Tavilla - Roman Kralik - Marie Roubalova
}

DOI: $10.18355 /$ XL.2019.12.04.19

\begin{abstract}
As Zeno of Elea disputed against multiplicity and local movement, so Kierkegaard disputed against the pretentious claim of his contemporaries "to go beyond faith" or simply to perform the movement of faith. To show how far people so called Christians were from genuine faith, Kierkegaard stresses in Fear and Trembling the exceptionality of Abraham's story, analysing the infinite stages through which the knight of faith must pass. The aim of this paper is to suggest possible analogies between Zeno's paradoxes and Kierkegaard's treatment of Genesis 22.
\end{abstract}

Key words: Kierkegaard, Zeno of Elea, paradox, Abraham, Fear and Trembling, knight of faith

\section{Sintesi}

Zenone di Elea ha confutato la molteplicità e il movimento. Dal canto suo, Kierkegaard intende contestare la pretesa avanzata dai suoi contemporanei di "andare oltre la fede" o semplicemente di compiere il movimento della fede. Per mostrare quanto i sedicenti cristiani siano in realtà alieni da una fede autentica, Kierkegaard enfatizza in Timore e tremore l'eccezionalità della vicenda di Abramo, considerando le infinite tappe attraverso le quali il cavaliere della fede deve passare prima di superare la prova. Si cercherà di suggerire, nelle pagine che seguono, possibili analogie tra i paradossi zenoniani e i dispositivi narrativi utilizzati da Kierkegaard nella riscrittura che egli fa di Genesi 22.

Parole chiave: Kierkegaard, Zenone di Elea, paradosso, Abramo, Timore e tremore, cavaliere della fede

Introduzione. Nel 1843, l'anno del suo esordio letterario, Kierkegaard portava in stampa ben sei volumi, tra i quali: Enten-Eller, Timore e tremore, La ripetizione. Con essi faceva il proprio ingresso in società la prima variopinta compagine di eteronimi (Victor Eremita, Johannes de Silentio, Constantin Constantius), alla quale si affiancava tuttavia, strategicamente, la produzione "edificante" dei Discorsi, firmata di suo pugno dall'autore (a questo proposito cf. Zalec, 2018a: 49).

Dietro la studiata frammentarietà e asistematicità della produzione kierkegaardiana, volta ad animare una sorta di teatro di maschere (cf. Amoroso, 1990) tra le quali il lettore è chiamato a riconoscere il proprio punto di vista e ad assumerlo consapevolmente - ponendo così fine all'anonimato e all'impersonalità, che Kierkegaard considerava i mali del proprio tempo (cf. Martín, 2017: 103) - è possibile rintracciare un'unità di contenuto, stante in ciò che Kierkegaard riconobbe essere il suo compito di scrittore: rettificare la definizione di cristiano (cf. Kierkegaard, 2006), ovvero spiegare cosa significasse in realtà essere cristiani a una moltitudine di sedicenti tali (Siclari, 2004: 303 e Tavilla, 2013: 548).

Kierkegaard ha escogitato una modalità di comunicazione originale per rendere i propri contemporanei e correligionari consapevoli dell'esigenza cristiana un'esigenza severa, perché infinita, fuori della misura umana, ben più onerosa delle pratiche religiose officiate dal clero nell'ambito di una cristianità ormai

XLinguae, Volume 12, Issue 4, October 2019, ISSN 1337-8384, eISSN 2453-711X 
istituzionalizzata, cui Kierkegaard rimprovera l'apostasia e la "carnevalizzazione" del cristianesimo (cf. Kierkegaard, 2001a; cf. anche Kierkegaard, 2016).

In aggiunta a ciò, Kierkegaard ha dovuto fronteggiare sul versante filosofico e culturale la volontà egemonica della filosofia idealista, specialmente quella di marca hegeliana, la quale pretendeva di "andare oltre" la fede, sancendo così il primato del concetto sul mito biblico (Tavilla, 2013: 545). Contro questa doppia deriva Kierkegaard ha preso posizione, impegnando se stesso, le sue sostanze e le sue non comuni doti letterarie. (Kralik, 2013: 443-451).

L'infinito nella sfera estetica $\mathbf{e}$ in quella etica. Tra i concetti su cui Kierkegaard ha maggiormente insistito per denunciare la bancarotta spirituale della propria epoca - tanto prodiga nel professare la fede cristiana quanto nell'affermare di averla superata - vi è l'infinito, cui il filosofo danese giunge sia per divisione che per composizione. È degno di nota come questo concetto si ritrovi opportunamente declinato nelle diverse sfere dell'esistenza individuate da Kierkegaard: l'estetico, l'etico e il religioso.

L'infinito "estetico" è l'infinito per composizione, e si esprime nella voracità bulimica che muove il seduttore ad accumulare sempre nuove esperienze erotiche, senza poter soddisfare mai il proprio desiderio. Questo perché il piacere di cui Don Giovanni non è mai pago non consiste nel possesso bensì nel processo della conquista. Il piacere del seduttore kierkegaardiano non è grossolano e sensuale ma sofisticato e cerebrale. Di contro al volgare edonista, l'esteta fugge la ripetizione, procacciandosi occasioni sempre nuove di godimento. In ciò risiede la sua raffinatezza ma anche la ragione profonda della sua condanna. Mentre infatti i sensi non hanno nozione che di piaceri finiti, l'immaginazione di cui il seduttore è preda non ha limite.

L'infinito "etico" è l'infinito per divisione. Esso coincide con la legge mosaica e gli innumerevoli precetti in cui essa si specifica e dai quali ogni uomo di buona volontà resta sopraffatto. Il legalismo giudaico è l'espressione di un'esigenza assoluta che vanifica ogni sforzo umano. Lo stadio etico trova la propria ipostatizzazione nella figura del marito, il quale, al contrario del seduttore, non fugge la ripetizione, anzi la cerca, trascorrendo la propria vita nel tentativo non meno disperato di rinnovare ogni giorno le promesse nuziali e di adempiere ai doveri che la sua posizione sociale gli impone. Malgrado gli sforzi di cui l'etico dà prova, esso culmina nel senso di colpa. Come già aveva ben visto Hegel, infatti, esiste un dissidio tra essere e dover essere che rende la vita morale irrealizzabile e dunque invivibile.

L'infinito religioso: la parabola del debitore insolvente. Kierkegaard ha enfatizzato l'eterogeneità qualitativa tra l'uomo e Dio, salvaguardando la trascendenza di quest'ultimo contro ogni forma di immanentismo e misticismo. Tra il creatore e la creatura egli riconosce l'esistenza di una distanza infinita, che non può essere tolta e che nemmeno l'incarnazione di Dio in Cristo ha il potere di revocare (Tavilla, 207: 561).

Kierkegaard paragona perciò la condizione di ogni cristiano a quella di un debitore insolvente cui è stato condonato un debito infinitamente grande (Pap. XI ${ }^{2} \mathrm{~A}$ 286; Pap. $\mathrm{XI}^{2} \mathrm{~A}$ 367). In questo si è forse ispirato all'episodio del Vangelo di Luca (7, 36-50), in cui il Cristo, ospite di Simone il fariseo, viene avvicinato da una peccatrice che si mette a baciargli i piedi e a cospargerli di olio profumato. Il fariseo dubita tra sé che Gesù sappia chi sia la donna, ma avendo intuito i suoi pensieri Gesù gli racconta la parabola dei due creditori: "Un creditore aveva due debitori: l'uno gli doveva cinquecento denari, l'altro cinquanta. Non avendo essi da restituire, condonò il debito a tutti e due. Chi dunque di loro lo amerà di più?".

Ora, il debito che ogni uomo ha contratto con Dio è talmente grande che il debitore, se anche lo volesse, non potrebbe comunque estinguerlo. Quel che conta 
però è che egli conservi riconoscente memoria del proprio creditore. E questo è per lui l'unico modo di mostrargli gratitudine: non dimenticare l'entità del debito che gli è stato rimesso.

La fede si riceve in virtù della grazia di cui l'uomo si dimostra capace solamente dopo essersi sforzato con tutto se stesso di imitare un modello che non potrà mai essere eguagliato. "Ora si potrebbe obiettare: 'che senso ha sforzarsi di eguagliare un modello che è infinitamente lontano e perciò irraggiungibile?' In realtà, è solo lo sforzo che l'uomo compie per conformare la propria vita alla sequela del Cristo sofferente sulla croce a renderlo consapevole dell'infinita distanza che lo separa dal Modello e dunque a renderlo parimenti cosciente dell'infinito valore della grazia che gli è stata elargita. Solo a questa condizione, a patto cioè che l'uomo sperimenti prima la propria insufficienza, la grazia diventa efficace" (Tavilla, 2017: 358). Perciò l'uomo non oltrepasserà mai la fede, e appunto nel diventare cosciente di ciò ottiene la prova della propria salvezza.

La corsa della fede. "Non ho certo raggiunto la mèta, non sono arrivato alla perfezione, ma mi sforzo di correre per conquistarla, perché anch'io sono stato conquistato da Gesù Cristo. Fratelli, io non ritengo di averla ancora conquistata. So soltanto questo: dimenticando ciò che mi sta alle spalle e proteso verso ciò che mi sta di fronte, corro verso la mèta, al premio che Dio ci chiama a ricevere lassù, in Cristo Gesù". Così scrive l'apostolo Paolo alla comunità di Filippi (Fil: 3,8-14), paragonando la fede alla corsa verso un traguardo, la perfetta conformità a Cristo, che l'apostolo dichiara però di essere ancora lontano dal raggiungere.

La pericope che abbiamo citato individua la possibile matrice scritturale dell'idea che la fede risieda in un movimento e che, mutatis mutandis, come il piacere estetico, anch'essa non consista nel possesso ma nella conquista. In altri termini, Kierkegaard pensa che la fede non sia un bene di cui il cristiano possa menar vanto, né tanto meno un'acquisizione storica, che possa essere trasmessa da una generazione alle altre, come accade al sapere tecnico-scientifico (a tale proposito cf. Kondrla, P., Durkova, E., 2018; vedasi anche Pavlikova, M., 2018), ma piuttosto un continuo processo di appropriazione, che ricomincia con ogni singolo individuo e non può dirsi concluso se non al termine dell'esistenza.

Sempre l'Apostolo scriverà a Timoteo, in punto di morte, "ho combattuto la buona battaglia, ho terminato la mia corsa, ho conservato la fede" (2Tim: 4,7). Ciò che una volta ci è appartenuto va infatti saputo mantenere, "poiché l'importante è non dimenticare, nell'entusiasmo della vittoria, di conservare ciò che si è conquistato, di proseguire ciò che si è intrapreso" (Høffding, 2015: 94).

"Ai giorni nostri - sentenzia polemicamente Johannes de Silentio, nell'Introduzione a Timore e tremore - non ci si ferma alla fede; si va oltre. [...] Non era così una volta; allora la fede era compito assegnato all'intera esistenza; perché, si pensava, l'attitudine a credere non si acquista in pochi giorni o in poche settimane. Quando il vecchio combattente, dopo aver sostenuto la sua buona battaglia e conservato la fede, giungeva alla fine, il suo cuore era ancora rimasto abbastanza giovane per non avere dimenticato l'angoscia e il tremore che erano stati disciplina della sua giovinezza, che l'uomo maturo aveva successivamente dominato, ma di cui nessuno si libera interamente, a meno che non si riesca ad andare 'oltre' il più presto possibile" (Kierkegaard, 2001b: 5).

Primo avvicinamento ad Abramo. Se il titolo dell'opera d'esordio, EntenEller, la più corposa che Kierkegaard abbia mai scritto, suona polemicamente antihegeliano, le alternative tra le quali il lettore è chiamato a scegliere conducono in realtà a un nulla di fatto. L'estetico mette capo alla noia, l'etico al senso di colpa, sintomi entrambi di una disperazione profonda che alligna alla radice dell'animo

XLinguae, Volume 12, Issue 4, October 2019, ISSN 1337-8384, eISSN 2453-711X 
umano - perché ognuno è disperato, pensa Kierkegaard, anche chi in apparenza non lo dà a vedere. Anzi, la forma più pericolosa di disperazione è proprio quella di chi ignora di essere disperato (la causa della disperazione sta nella presunzione dell'uomo di bastare a se stesso, di negare il proprio rapporto costitutivo con la potenza che lo ha posto).

Non "o l'uno o l'altro", "o questo o quello", come a prima vista l'autore sembrerebbe suggerire, in contrasto con la tendenza della filosofia hegeliana a operare sintesi di contrari, ma piuttosto "né l'uno né l'altro", "né questo né quello" è il senso ultimo dell'aut-aut kierkegaardiano. Al termine di Enten-Eller il lettore si ritrova al punto di partenza, senza essersi mosso di un passo, come se fosse entrato in un labirinto magico con due ingressi che conducono entrambi allo stesso vicolo cieco. La percezione che se ne ricava è quella di un movimento illusorio, un girare a vuoto che disattende ogni promessa di progressione dialettica. Kierkegaard ha infatti sostituito il concetto di linearità con quello di segmento, il sistema con il frammento, la "briciola" filosofica; occorre dunque compiere un movimento a ritroso, lungo il binario morto che è stato erroneamente imboccato, prima di poter ristabilire un rapporto autentico con la verità.

L'unico sentiero percorribile è quello della fede, che in tre giorni ha condotto Abramo sul monte Moriah - il luogo dove si sarebbe dovuto consumare il sacrificio di Isacco (il tema del sacrificio in Kierkegaard è stato recentemente trattato in Zalec, 2017). Sul cammino intrapreso dall'anziano patriarca e sugli stati d'animo (Stemning) che lo accompagnano, Kierkegaard si concentra nella prima sezione di Timore e tremore.

I movimenti della fede. "Ma veramente ognuno dei miei contemporanei è capace di compiere i movimenti della fede?" (Kierkegaard, 2001b: 37). L'interrogativo retorico che Johannes de Silentio lancia provocatoriamente al lettore intende smascherare l'ipocrisia e la superficialità di quanti affermano di essere andati "oltre" la fede. L'intento di Kierkegaard è quello di mostrare quanto sia difficile compiere l'atto di fede e, per converso, quanto sia facile ingannarsi in merito allo stato della propria fede, ovvero quanto sia facile illudersi di possederla quando in realtà non la si è mai raggiunta. Al limite questo potrebbe anche essere, "ma non è permesso a nessuno di far credere agli altri che la fede ha poca importanza o che è una cosa facile, quando, invece, essa è la più difficile di tutte" (Kierkegaard, 2001b: 61).

L'operazione che Kierkegaard realizza ha un risvolto comunicativo complesso, in quanto consiste nel demistificare ciò a cui comunemente ci si riferisce col termine fede o vita religiosa e nel riqualificare queste esperienze in modo da restituire loro la ricchezza di significato che la nostra epoca ha smarrito. "L'epoca nostra organizza una vera e propria liquidazione nel mondo delle idee come in quello degli affari. Ogni cosa può essere comprata a prezzi tanto bassi, che è possibile domandarsi se alla fine ci saranno ancora acquirenti" (Kierkegaard, 2001b: 3). Si tratta dunque di "rivalutare" la fede - per continuare con la metafora monetaria cara a Kierkegaard - attraverso una serie di strategie narrative "deflazionistiche" su cui vale la pena soffermarsi. Iben Damgaard ha parlato a questo proposito di "giocare allo straniero con ciò che è noto" (Damgaard, 2008: ), alludendo alla volontà di Kierkegaard di dare nuova linfa a personaggi e luoghi biblici che il lettore conosce attraverso una lettura stereotipata e conformista, in capace ormai di produrre edificazione.

È possibile distinguere alcune tecniche di cui Kierkegaard si avvale mentre riscrive l'episodio del sacrificio di Isacco, segnatamente: a) la mise en abyme; b) la dicotomia c) la relatività del punto di vista d) "il fermo immagine". Tutti questi accorgimenti sortiscono un effetto distanziante, che consente di mettere in risalto la grandezza di Abramo e la paradossalità della fede, la sua inaccessibilità all'uomo 
comune. Osserviamo poi che tali tecniche appartengono al repertorio dei paradossi di Zenone di Elena, al quale Kierkegaard potrebbe forse essersi ispirato.

I paradossi zenoniani contro il movimento e la molteplicità. Zenone di Elea (IV a.C.) è noto per aver elaborato alcune argomentazioni per assurdo tese a confutare la molteplicità e il movimento (cf. Fano, 2013). Il paradosso della dicotomia afferma che per poter percorrere uno spazio, occorre prima giungere alla metà e prima ancora alla metà della metà $\mathrm{e}$, così via all'infinito. Non è possibile tuttavia percorrere uno spazio infinito in un tempo finito.

Il secondo paradosso ipotizza una gara di velocità tra Achille "piè veloce" e la tartaruga, animale proverbialmente noto per la sua lentezza. Concesso alla tartaruga un certo margine di vantaggio, l'argomentazione mostra, contro ogni evidenza empirica, che non solo Achille non riuscirà mai a superarla, ma nemmeno sarà in grado di raggiungerla, perché quando Achille si troverà nel punto da cui la tartaruga è partita, questa sarà avanzata di una grandezza, seppur minima, e così via, la distanza tra i due concorrenti tenderà a rimpicciolirsi all'infinito ma non sarà mai colmata.

L'argomento della freccia consiste nello scomporre la traiettoria che una freccia descrive dall'arco al bersaglio in infiniti istanti. Ad ogni istante, essa occupa uno spazio pari alla propria lunghezza, risultando di fatto sempre immobile.

L'argomento delle masse nello stadio afferma che due masse, che si muovono alla stessa velocità in direzione opposta, percorrono la stessa distanza in un tempo dato e, nella metà di esso, a seconda del punto di osservazione che scegliamo di assumere.

Contro la molteplicità Zenone afferma, poi, che se esiste una certa quantità essa è finita, ovvero pari a se stessa e, al tempo stesso, infinita. Stando alla concezione aritmo-geometrica dei pitagorici, infatti, ogni unità corrisponde a un punto fisico nello spazio. L'esistenza di molteplici unità è possibile a condizione che tra un punto e un altro vi sia un intervallo minimo, un terzo punto quindi, che impedisca ai primi due di collassare l'uno sull'altro. Ma così facendo tra un punto e un altro dovrà ammettersi sempre un'altra unità, all'infinito.

Il paradosso degli antichi e quello dei moderni. I paradossi zenoniani sono tali nella misura in cui smentiscono l'esperienza sensibile. Etimologicamente, il termine paradosso $\left(\pi \alpha \rho \alpha ́ \delta\right.$ o $\left.\varsigma_{o}\right)$ significa ciò che va "contro" ( $\left.\pi \alpha \rho \alpha-\right)$ l'opinione comune $(\delta o ́ \xi \alpha)$. E l'opinione si fonda appunto sull'esperienza fenomenologica, la quale testimonia la realtà del movimento e della molteplicità. Ad essa si oppone la verità di ragione, fondata sul principio di non contraddizione.

La dimostrazione per assurdo di cui Zenone si serve per confutare il movimento e la molteplicità prevede che le tesi degli avversari siano accolte in premessa come vere per poi mostrare che esse conducono a conclusioni assurde, ovvero auto-contraddittorie, e dunque irreali.

La messa in evidenza della cifra di paradossalità che contraddistingue il cristianesimo e la rivendicazione della sublimità della fede, della sua irriducibilità a sistema, rientrano programmaticamente tra le finalità dell'opera di Kierkegaard. Per il filosofo danese paradosso significa però qualcosa di diverso dal "contro-senso" zenoniano, al punto, diremmo, che se è parso giusto distinguere, nell'ambito della riflessione kierkegaardiana sul tragico, tra il tragico antico e il tragico moderno, sembra lecito, allo stesso modo, pensare di poter distinguere tra il paradosso degli antichi, segnatamente il paradosso zenoniano, e il paradosso "dei moderni" - come avrebbe detto Kierkegaard - cioè il paradosso cristiano.

Mentre il paradosso zenoniano vuole restaurare il principio di non contraddizione e l'identità della verità con se stessa, il paradosso cristiano risiede, al contrario, nell'identità tra la verità e la contraddizione (Lc: 2,34$)$.

XLinguae, Volume 12, Issue 4, October 2019, ISSN 1337-8384, eISSN 2453-711X 
Questa concezione risente inoltre della theologia crucifixa di Lutero, il cui nucleo centrale consiste nella rivelazione di Dio sub contraria specie (Regina, 2018: 78). La stessa teoria kierkegaardiana della comunicazione indiretta sembra improntata dalla contraddizione tra esteriorità ed interiorità che si rivela nel mistero della croce.

La tecnica della mise en abyme. Consideriamo ora le strategie messe in atto da Kierkegaard in Timore e tremore, a partire dalla mise en abyme - l'equivalente del paradosso zenoniano delle infinite unità. Tale "tecnica" consiste nel riconoscere che, via via, una misura in più viene ad aggiungersi tra sé e la verità, ottenendo l'effetto di un allontanamento sempre maggiore della verità medesima proprio quando, esplicitamente, si fa il possibile per introdurci ad essa. Il "distanziamento" caratterizza la produzione pseudonimia kierkegaardiana, specialmente i testi citati all'inizio.

In Timore e tremore esso risponde a un preciso schema che simula il giuoco a incastro delle scatole cinesi. La storia di Abramo rappresenta il nucleo drammatico dell'opera, ma prima di giungere ad essa Kierkegaard vuole che il lettore attraversi in successione una sequenza di cornici la cui funzione non è solo quella di introdurci alla vicenda, ma soprattutto quella di farci apprezzare la distanza antropologica e spirituale che ci separa dai suoi protagonisti. L'editore dell'opera, Johannes de Silentio (1 livello) riferisce di un uomo (2 livello), il quale trascorre una vita intera nel vano tentativo di comprendere il senso della figura di Abramo (3 livello).

$\mathrm{Si}$ noti come questo effetto risulti amplificato dalla scelta operata da Kierkegaard di scandire in tre momenti distinti la meditazione che l'uomo di cui si parla conduce su Genesi 22. "C'era una volta un uomo che durante la sua infanzia aveva udito la bella storia di Abramo messo alla prova da Dio...". Riprende al paragrafo successivo: "In età matura, rilesse con cresciuto stupore quel racconto, perché la vita aveva separato quanto era unito nella pia semplicità dell'infanzia". La parte evidenziata in corsivo sembra costituire un'indicazione di metodo. Si tratta, infatti, per Kierkegaard, di dilatare gli spazi, di ristabilire le giuste distanze tra i suoi contemporanei e gli eroi biblici per mezzo dei quali l'umanità si illude di essere entrata una volta per tutte in confidenza con Dio. Continua poi al paragrafo successivo: "Man mano che egli invecchiava, il suo pensiero tornava più di frequente a quella storia, con una passione sempre più grande; e tuttavia, la comprendeva sempre meno" (Kierkegaard, 2001b: 9).

La tecnica della dicotomia. Per dimostrare che l'atto di fede è fuori della nostra portata o comunque assai difficile da realizzarsi, Johannes de Silentio, proprio come Zenone di Elea, procede per dicotomie. Il movimento della fede viene scomposto in due momenti, la rassegnazione infinita e l'atto di fede vero e proprio, suscettibili di essere ulteriormente distinti in molteplici approssimazioni, in un regresso che tende all'infinito.

"La rassegnazione infinita è l'ultimo stadio precedente la fede; di modo che chiunque non ha fatto quel movimento non ha fede" (Kierkegaard, 2001b: 54). Prima di poter compiere l'atto di fede un uomo deve saper rinunciare al mondo. Ciò non significa tuttavia che chi opera tale rinuncia sia per ciò stesso un uomo di fede. "La rassegnazione non implica la fede", ovvero "per rassegnarsi non è necessaria la fede" come invece vorrebbe l'opinione comune (Kierkegaard, 2001b: 56).

Ciò che importa notare qui è come il primo movimento che la fede esige sia la rinuncia, e come già quest'atto, che pur non essendo ancora l'atto della fede è tuttavia propedeutico ad esso, non sia alla portata di tutti. Per quanto, infatti, l'atto della rinuncia rimanga umanamente più comprensibile dell'atto di fede, esso richiede una forza d'animo non comune. Al punto che la rinuncia potrebbe assorbire tutte le energie di cui un individuo disponga. " $\mathrm{Ci}$ vuole un coraggio proprio umano per rinunciare a tutta la temporalità" (Kierkegaard, 2001b: 57). Del resto, osserva lo 
pseudonimo, la rinuncia di cui si parla non riguarda il superfluo di cui ciascuno fa volentieri a meno, ma il valore più alto che l'uomo incontri nel secolo, forse addirittura ciò che il secolo reputa essere dovere dell'uomo amare. "Perché - qui Johannes de Silentio parla del giovane cui Gesù rivolse l'invito ad abbandonare tutti i suoi beni - se egli ha ceduto i suoi beni perché ne era stanco, vuol dire che la sua rassegnazione lascia molto a desiderare" (Kierkegaard, 2001b: 57s.). Nemmeno Socrate, si noti, è mai giunto a compiere il movimento infinito che precede quello della fede. Infatti "Egli ha compiuto il movimento dell'infinito dal punto di vista intellettuale. La sua ignoranza è l'infinita rassegnazione. Questo compito è già sufficientemente grande per le forze umane, benché oggi lo si sdegni; ma bisogna prima averlo eseguito, bisogna prima che l'Individuo si sia consunto nell'Infinito, perché egli giunga al punto dal quale può sorgere la fede" (Kierkegaard, 2001b: 84).

La relatività del punto di vista. Un'altra tecnica narrativa di cui Kierkegaard si avvale consiste nel moltiplicare i punti di vista e nel considerare le possibili varianti, persino le più aberranti, che il testo biblico non necessariamente autorizza ma alle quali nondimeno si presta.

Per effetto di questo studiato ipertrofismo, si direbbe che il viaggio di Abramo sul monte Moriah non sia percorribile una seconda volta (quanto è vero che non ci si può bagnare due volte nello stesso fiume, e forse nemmeno una, stando all'adagio eracliteo citato al termine di Timore e tremore). La sua durata è finita, pari a tre giorni di cammino (tanti sono quelli di cui la Genesi dà notizia), e al tempo stesso infinita, data da innumerevoli "moduli" di tre giorni (quante sono le volte che il lettore tornerà a meditare la vicenda di Abramo). In effetti, Johannes de Silentio lascia intendere che le varianti del testo biblico possono essere più di quattro, cioè più di quelle effettivamente presentate al lettore: "così e in vari altri modi, rifletteva su quell'avvenimento l'uomo del quale parlavamo" (Kierkegaard, 2001b: 14).

Ognuna delle riscritture che vengono ipotizzate si conclude con la perdita della fede da parte di Abramo (I, II e III variante) o di Isacco (IV variante), a conferma di quanto sia facile illudersi di possedere la fede senza che, in realtà, la si abbia mai raggiunta.

"C'è chi interpreta la storia di Abramo in un altro modo. Si celebra la grazia di Dio che concedette Isacco per la seconda volta e in tutta la storia non si scorge altro che una prova per Abramo. Una prova: è dir molto e dir poco. Detto e fatto. S'inforca Pegaso, in un batter d'occhio si arriva a Moriah e si vede subito l'ariete" (Kierkegaard, 2001b: 62). Uno sguardo frettoloso e superficiale genera la percezione distorta che il tempo della prova si esaurisca nel tempo della lettura. Johannes de Silentio avverte perciò: "Vorrei [...] ricordare che il viaggio durò tre giorni e una buona parte del quarto; e quei tre giorni e mezzo, io li farei durare infinitamente più a lungo delle migliaia di anni che ci separano dal patriarca" (Kierkegaard, 2001b: 63).

La tecnica del "fermo immagine". La dilatazione del tempo, funzionale alla percezione della "distanza" che separa l'eroe biblico dai suoi inseguitori, e presunti "figli nella fede", viene procurata da ultimo grazie alla tecnica del "fermo immagine", procedimento affine al paradosso zenoniano della freccia.

Se nel ragionamento del filosofo di Elea, la parabola di un dardo veniva scomposta in infiniti istanti, per provare che ad ogni istante esso è immobile, e dunque non potrà mai raggiungere il bersaglio, qui Kierkegaard sottopone la salita di Abramo sul monte Moriah a un trattamento analogo: individuare e isolare ogni gesto compiuto da Abramo, conferendo ad esso un valore icastico sul quale il lettore è invitato a meditare. Ma se ogni gesto, come ogni parola, ogni singolo yod deve essere meditato, nessuno potrà mai dire di aver compreso Abramo fino in fondo, né tanto meno potrà vantarsi di essere andato oltre, perché in realtà il lettore incontrerà sempre qualcosa su

XLinguae, Volume 12, Issue 4, October 2019, ISSN 1337-8384, eISSN 2453-711X 
cui soffermarsi, e nel far ciò starà, etimologicamente, "fermo per un poco", quanto basta per non poter mai dire di aver concluso il suo cammino.

Johannes de Silentio ha reso efficacemente il senso di un movimento "congelato" nella paratassi delle quattro atmosfere proemiali, dove all'incedere torrenziale della prosa kierkegaardiana subentra una sintassi frammentata, fatta di periodi brevi, scanditi da una punteggiatura insistita, che dà forma a una sequenza telegrafica di quadri statici. Valgano da esempio le seguenti righe: "Era mattino presto. Abramo si levò, abbracciò Sara, la fidanzata della sua vecchiaia, e Sara dette un bacio a Isacco che l'aveva preservata dalla vergogna, lui, l'orgoglio suo e speranza sua per tutta la posterità. Cavalcarono in silenzio. Lo sguardo di Abramo rimase fisso a terra fino al quarto giorno. Allora, vide all'orizzonte la montagna di Moriah. Abbassò di nuovo lo sguardo. Preparò l'olocausto in silenzio e legò Isacco. In silenzio estrasse il coltello. Allora scorse il capro provveduto da Dio. Lo sacrificò e tornò indietro. Da quel giorno Abramo fu vecchio; non poteva dimenticare quel che Dio aveva preteso da lui. Isacco continuò a crescere. Ma l'occhio di Abramo s'era fatto cupo; non vide mai più la gioia" (Kierkegaard, 2001b: 12).

Solvitur ambulando. Diogene di Sinope e il movimento della ripetizione. $L a$ ripetizione si apre con la celebre immagine di Diogene il cinico, il quale si oppose "a chi diceva che il movimento non esiste" levandosi in piedi e mettendosi a camminare (Diogene Laerzio, 2005: 647). Com'è noto questa prova non possiede agli occhi degli eleati alcuna forza contro-argomentativa per il semplice fatto che l'esperienza, cui Diogene fa appello, non ha alcun fondamento logico, ma è appunto l'illusione di cui gli ignoranti si pascono.

Alla maniera di Diogene, Constantin Constantius, lo pseudonimo di turno, intende sperimentare la possibilità concreta di una "ripetizione", sforzandosi di realizzarla fattualmente. Partirà, quindi, alla volta di Berlino, meta di un suo precedente viaggio, per vedere se riuscirà a rivivere la stessa esperienza vissuta durante il suo primo soggiorno laggiù. L'esito di questo esperimento sarà, malgrado gli accorgimenti adottati da Constantin e i reiterati sforzi posti in essere dallo stesso, fallimentare. Lo pseudonimo dovrà perciò concludere di non essere all'altezza di un simile "movimento religioso" (Kierkegaard, 1996: 84; Tavilla, 2012: 76).

È alquanto significativo che Kierkegaard invochi l'esempio di Diogene. Il filosofo cinico rappresenta la rivolta del senso comune contro l'astrusità della filosofia, e già questo basta a porlo sulla stessa lunghezza d'onda del filosofo danese. Ma il contesto all'interno del quale l'episodio si inserisce lo arricchisce anche di un altro significato: contro le pretesa della filosofia di poter compiere qualsiasi movimento con il pensiero e contro la riduzione del cristianesimo a dottrina, Kierkegaard esige che la professione di fede sia sempre seguita dalla testimonianza (cf. Máhrik, T., Pavlíková, M., Root, J., 2018: 52).

La ripetizione corrisponde al movimento della fede, giacché vale per essa ciò che Kierkegaard affermava della fede: non è possibile se non in forza dell'assurdo. Ora, accade che sia facile ingannarsi sulla praticabilità di questo movimento, almeno fin tanto che non ci si sia sforzati di metterlo in opera. Per questo Constantin Constantius si incarica di mostrare l'inattuabilità pratica di un simile movimento, unendo alla dialettica contro-intuitiva di Zenone il sano pragmatismo di Diogene.

L'infinito regresso della fede. Abbiamo considerato le strategie portate avanti da Kierkegaard in Timore e tremore allo scopo di delegittimare la pretesa della filosofia hegeliana di oltrepassare la fede, relegando quest'ultima al rango di verità minore, riservata "alle nature più incolte" (Kierkegaard, 2001b: 40).

La posizione di Kierkegaard - in linea con l'insegnamento dell'apostolo Paolo, il quale presenta la vita cristiana come una corsa in cui la fede deve essere conservata fino alla fine - è che la fede è una prova, che "tutta la vita è una prova" 
(Kierkegaard, 2001b: 62), e che neppure Abramo è andato al di là della fede, sebbene sia vissuto per centrotrent'anni. L'atto di fede è un fatto prodigioso, per il quale l'uomo ripete (at gjentage), ovvero ottiene per la seconda volta (at tage igjen), in forza dell'assurdo, ciò a cui ha rinunciato in precedenza grazie al movimento dell'"infinita rassegnazione".

$\mathrm{Ci}$ è parso possibile accostare gli espedienti narrativi adottati da Kierkegaard ai paradossi zenoniani contro il movimento e la molteplicità. In particolare, si è visto come Kierkegaard cerchi di operare costantemente un distanziamento tra il lettore e il modello cui quest'ultimo è chiamato a conformarsi, e a tale scopo, si avvalga di procedimenti molto simili a quelli messi in atto dal filosofo di Elea, come la dicotomia, la moltiplicazione degli enti (mise en abyme), la relatività del punto di vista, e il fermo immagine. Tutti questi dispositivi sono finalizzati a produrre un effetto distanziante, che culmina in un regressus ad infinitum.

Se Zenone intende dimostrare agli avversari di Parmenide che Achille "piè veloce" non raggiungerà mai la tartaruga, Kierkegaard vuole provare ai suoi contemporanei, sedicenti cristiani, che l'intervallo che li separa dall'autentica fede cristiana è infinito e, come tale, incolmabile. La tesi kierkegaardiana dell'insuperabilità della fede si basa sulla teologia luterana della croce e presuppone l'infinita differenza qualitativa tra Dio e l'uomo. Resta tuttavia compito di quest'ultimo sforzarsi di eguagliare il Modello - Cristo crocifisso - ben sapendo, però, che non potrà mai raggiungerlo, perché solo attraverso questo sforzo prenderà coscienza dell'infinita distanza che lo separa da Lui e solo così potrà apprezzare il valore infinito della grazia che gli è stata elargita.

This article was published with the support of the Slovak Research and Development Agency under the contract No. APVV-17-0158.

\section{Bibliographic references}

AMOROSO, L. 1990. Maschere kierkegaardiane. Torino: Rosenberg \& Sellier. ISBN 9788870114089.

DAMGAARD, I. 2008. At lege fremmed med det kendte. Copenhagen: Anis. ISBN 978-8774574637.

DIOGENE LAERZIO 2005. Vite e dottrine dei più celebri filosofi. Milano: Bompiani. ISBN 978-88-452-3301-4.

FANO, V. 2013. I paradossi di Zenone. Roma: Carocci. ISBN 978-88-430-6267-6.

HØFFDING, H. 2015. Kierkegaard umanista, tr. it. di I. Tavilla. Roma: Castelvecchi. ISBN 978-88-694-4416-6.

KIERKEGAARD, S. 2016. Io voglio onestà. Contro le menzogne del cristianesimo ufficiale, Roma: Castelvecchi. ISBN 978-8869445538.

KIERKEGAARD, S. 2006. Sulla mia attività di scrittore, Pisa: ETS. ISBN 978-88467-1663-7.

KIERKEGAARD, S. 2001a. L'istante, tr. it. di A. Gallas, Genova: Marietti. ISBN 978-88-211-6119-3.

KIERKEGAARD, S. 2001b. Timore e tremore, tr. it. di F. Fortini e K. Montanari Guldbradsen, Milano: Mondadori. ISBN 978-88-04-67089-6.

KIERKEGAARD, S. 1996. La ripetizione, tr. it. di D. Borso. Milano: BUR. ISBN 978-8817057950.

KONDRLA, P. - DURKOVA, E. 2018. Interpersonal relations in ethics of science and technologies Communications - Scientific Letters of the University of Zilina 20 (1PartA), pp. 45-50.

KRALIK, R. 2013. The Reception of Soren Kierkegaard in Czech Language Writings. In: Filosoficky casopis, vol. 61, n. 3, pp. 443-451. ISSN 0015-1831.

KRALIK, R. 2013. Kierkegaarduv Abraham. Ljubljana: KUD Apokalipsa. ISBN 978-

XLinguae, Volume 12, Issue 4, October 2019, ISSN 1337-8384, eISSN 2453-711X 
961-6894-30-2.

MAHRIK, T., - PAVLIKOVA, M., - ROOT, J. 2018. Importance of the incarnation in the works of C.S. Lewis and S. Kierkegaard. In: European Journal of Science and Theology, vol. 14, n. 2, pp. 43-53.

MARTÍN, J. G. 2017. The category of the single individual in Kierkegaard. In: European Journal of Science and Theology, vol. 13, n. 3, pp. 99-108. ISSN 18410464.

PAVLIKOVA, M. 2018. The power of modern technologies in the fiction of Don DeLillo. In: Communications - Scientific Letters of the University of Zilina, 20 (1Part A), pp. 57-60.

REGINA, U. 2018. Lutero e Kierkegaard. Per il ripristino della cristianità. In: Kierkegaard, S. L'immutabilità di Dio, tr. it. di U. Regina, Brescia: Morcelliana, pp.67-99. ISBN978-88-372-3255-9.

SICLARI, A. 2004. L'itinerario di un cristiano nella cristianità. Milano: Franco Angeli. ISBN 978-88-464-5774-5.

TAVILLA, I. 2017. Inconoscibilità di Dio e interiorizzazione della fede nella filosofia di Søren Kierkegaard. In: Revista Portouguesa de Filosofia, vol. 73, n. 2, pp. 479-506. ISSN 0870-5283 e ISSN 2183-461X.

TAVILLA, I. 2013. Comunicazione d'esistenza e paradigma biblico in Søren Kierkegaard. In: Rivista di Filosofia Neo-Scolastica, anno CV, nn. 3-4, pp. 545-555. ISSN 00356247 e ISSN 18277926.

TAVILLA I. 2012. Senso tipico e profezia. Verso una definizione del fondamento biblico della categoria di Gjentagelse. Milano: Mimesis. ISBN 978-88-575-1492-5.

ZALEC, B. 2018a. Kierkegaard's method. Edification and moral grammar of Christian values. In: European Journal of Science and Theology, vol. 14, n. 6, pp. 4754. ISSN 1841-0464.

ZALEC, B. 2017. The relevance of Kierkegaard for political thought. Violence and the divine command. In: European Journal of Science and Theology, vol. 13, n. 3, pp. 75-87. ISSN 1841-0464.

Words: 5695

Characters: 37292 (20,72 standard pages)

Dr. Igor Tavilla, $\mathrm{PhD}$.

Dipartimento di Discipline Umanistiche

Sociali e delle Imprese Culturali - University in Parma

Via D’Azelio, 85 - 43100 Parma

Italy

igortavi@libero.it

Prof. Dr. Roman Kralik, ThD.

Faculty of Arts

Constantine the Philosopher University in Nitra

Hodzova 1,949 01 Nitra

Slovakia

rkralik@ukf.sk

ThDr. Marie Roubalová, ThD.

Hussite Theological Faculty

Charles University in Prague

Pacovská 350/4, 14000 Praha

Czech Republic

marie.roubalova@htf.cuni.cz 